\title{
Significance of serum neural precursor cell-expressed developmentally downregulated protein 9 in melanoma
}

\author{
KAYHAN ERTURK, FARUK TAS, MURAT SERILMEZ, ELIF BILGIN and DERYA DURANYILDIZ \\ Department of Medical Oncology, Institute of Oncology, University of Istanbul, Istanbul 34093, Turkey
}

Received July 26, 2017; Accepted October 27, 2017

DOI: $10.3892 / \operatorname{mco} .2017 .1493$

\begin{abstract}
Neural precursor cell-expressed developmentally downregulated protein 9 (NEDD9) is a promoter for various cellular functions that result in tumorigenesis. The aim of the present study was to analyse the serum levels of NEDD9 in melanoma patients in order to evaluate its prognostic, predictive and diagnostic value. Data from 112 melanoma patients were retrospectively analyzed and ELISA assays were used to measure serum NEDD9 concentration. The median serum NEDD9 levels of the patients were significantly higher compared with those of the controls. Serum NEDD9 was not found to be associated with any of the clinicopathological parameters, and was also not found to be prognostic for survival in melanoma. Therefore, serum NEDD9 may be of diagnostic value in melanoma, but its usefulness in prognosis remains controversial. The important role of NEDD9 in tumor angiogenesis necessitates efforts to elucidate its interactions with the tumor microenvironment and its potential as a therapeutic target for malignancies.
\end{abstract}

\section{Introduction}

The Crk-associated substrate (Cas) family comprises four non-catalytic scaffolding proteins (NEDD9/HEF1/CAS-L, BCAR1/p130Cas, EFS/Sin, and HEPL/CASS4) that mediate the cell cycle, survival, migration/chemotaxis, apoptosis, differentiation and cell attachment (1-6). The Cas proteins have been thoroughly investigated, and even mildly overexpressed levels of these proteins have been found to be correlated with poor survival, resistance to chemotherapy and metastasis in malignancies such as melanoma, lung cancer, glioblastoma, and breast cancer. These proteins have not only been associated with cancer, but they have also been reported to be associated

Correspondence to: Dr Kayhan Erturk, Department of Medical Oncology, Institute of Oncology, University of Istanbul, Capa, Fatih, Istanbul 34093, Turkey

E-mail: kayhanerturk@gmail.com

Key words: neural precursor cell-expressed developmentally downregulated protein 9, melanoma, serum, prognostic, diagnostic with other non-malignant conditions, including polycystic kidney disease (7).

Neural precursor cell-expressed developmentally downregulated protein (NEDD9) interacts with novel SH2-containing protein family scaffold proteins and the adaptor proteins SHC and GRB2 via its C-terminal domain, and mediates the communication between receptor tyrosine kinases and integrins, so that receptors, such as T-cell, B-cell and integrin receptors, send upstream activation signals. Subsequently, focal adhesion kinase (FAK) and the Src and ABL families of kinases are activated and they, in turn, phosphorylate NEDD9 substrate domain even more extensively, which provides multiple binding sites, i.e., Y189, Y317 and Y279, for downstream effectors. FAK phosphorylation of the DYDY motif in the NEDD9 C-terminal generates a binding site for Src kinase, which enables NEDD9 to operate in migration and other signaling functions (7). Furthermore, Y189 phosphorylation by FAK and Src kinases is involved in focal adhesion. Aurora-A kinase phosphorylates S296; thus, proteasomal degradation of NEDD9 ensues, and cell dissemination and the cell cycle are regulated.

NEDD9 is not only activated by FAK and Src kinases, but also maintains incessant activation of these kinases. NEDD9 connects tumor growth factor- $\beta /$ SMAD and Rho-actin-SRF signals, thus participating in tumorigenesis by coordinating the expression of relevant genes. It also activates matrix metalloproteinases (MMPs) and mediates actin branching and lamellipodia formation. NEDD9 downregulates E-cadherin expression by upregulating certain transcription factors, such as SLUG and SNAIL, modulates the Src-dependent E-cadherin removal from junctions and furthers invasion by degradation of the basal membrane through active MMP2 production (7).

In brief, NEDD9 brings protein complexes together to promote various cellular functions that result in tumorigenesis and stimulation of tumor cell proliferation, migration, and genomic instability. The aim of the present study was to analyse the level of NEDD9 in the serum of melanoma patients in order to evaluate its prognostic, predictive and diagnostic value in melanoma.

\section{Patients and methods}

Patients and treatment. The data of 112 melanoma patients, who had been treated and followed up between November 
Table I. Values of serum assay NEDD9 levels in melanoma patients and healthy controls.

\begin{tabular}{|c|c|c|c|c|c|}
\hline \multirow[b]{2}{*}{ Assay } & \multicolumn{2}{|c|}{ Patients ( $\mathrm{n}=112$ ) } & \multicolumn{2}{|c|}{ Controls $(n=43)$} & \multirow[b]{2}{*}{ P-value } \\
\hline & Median & Range & Median & Range & \\
\hline NEDD9 (ng/ml) & $3,784.02$ & $1,528.09-7,367.17$ & $2,149.03$ & $54.88-7,505.40$ & $<0.001$ \\
\hline
\end{tabular}

NEDD9, neural precursor cell-expressed developmentally downregulated protein 9.

2013 and March 2015, were included in the present study. Neither chemotherapy nor radiotherapy were administered to the patients over the last 6 months prior to inclusion. The American Joint Committee on Cancer staging system was used to determine the stage of the disease (8). Patients were assessed using clinical history, physical examination and a series of blood tests, such as lactate dehydrogenase and complete blood count, prior to the onset of treatment. To patients with an Eastern Cooperative Oncology Group performance status score of $\leq 2$ and good blood chemistry test results, treatment comprising interferon- $\alpha$, temozolamide, dacarbazine and cisplatin was administered in the outpatient clinic. In accordance with the stage of their disease, the patients received radiotherapy. Immunotherapy agents, such as pembrolizumab and nivolumab, and targeted therapy agents, such as vemurafenib/cobimetinib and dabrafenib/trametinib, were used for metastatic or unresectable disease. Clinical, laboratory and radiological assessments were performed every 8 weeks during chemotherapy and every 12 weeks after treatment completion. The revised Response Evaluation Criteria In Solid Tumors, version 1.1., were used to determine response to treatment (9). A total of 43 age- and sex-matched healthy controls were also included in the analysis. Informed consent was obtained from all patients and the study was reviewed and approved by the local ethics committee.

Sample collection. Serum samples were collected from treatment-naïve patients on first admission and after centrifugation they were stored at $-20^{\circ} \mathrm{C}$. A double antibody sandwich ELISA kit was used to determine the level of NEDD9 (cat. no. YHB3351; Shanghai YeHua Biological Technology Co, Ltd., Shanghai, China) in the samples, according to the manufacturer's instructions. Serum samples and standards were added to the wells that had been pre-coated with human NEDD9 monoclonal antibody. Streptavidin-horseradish peroxidase (HRP) and biotinylated-Fab monoclonal capture antibody conjugates were applied to form immune complexes and were then left to incubate at $37^{\circ} \mathrm{C}$ for $1 \mathrm{~h}$. Unbound streptavidin-HRP was washed away, and then a colorless chromogen solution was added and incubated at $37^{\circ} \mathrm{C}$ for $10 \mathrm{~min}$ (protected from light). The colorless solution turned blue, and the intensity of this color change was proportional to the amount of NEDD9 in the sample. The reaction was terminated by an acidic stop-solution and the color turned yellow. The end product was measured by an automated ELISA reader (ChroMate ${ }^{\circledR} 4300$ microplate reader; Awareness Technology Inc., Palm City, FL, USA) at $450 \mathrm{~nm}$. The results were expressed as $\mathrm{ng} / \mathrm{ml}$.
Statistical analysis. The statistical calculations were performed using SPSS software, version 21.0 (IBM Corp., Armonk, NY, USA). Continuous variables were divided using median values as cut-offs. The Mann Whitney U-test was used to analyze differences between groups with non-parametric data distribution. Survival was calculated from the date of first admission to the hospital to death from any cause or to the last contact with the patient or any family member. The survival time was analyzed by the Kaplan-Meier method and the differences in survival were assessed using log-rank statistics. A P-value $\leq 0.05$ was considered to indicate statistically significant differences.

\section{Results}

Patient characteristics. The median age at the diagnosis of the 112 patients was 52 years (range, 16-85 years), with a male predominance $(62 \%)$. Truncal lesions were observed in $55 \%$ and metastatic disease in $61 \%$ of the patients, with M1c disease in $72 \%$ of the cases. The baseline serum NEDD 9 levels of the patients were significantly higher compared with those of the healthy controls (median values: 3,784.02 vs. $2,149.03 \mathrm{ng} / \mathrm{ml}$, respectively; $\mathrm{P}<0.001$ ) (Table I). None of the known clinical parameters, such as age, site of lesion, lymph node involvement, stage, lactate dehydrogenase level, sex, histology, Breslow thickness, Clark invasion level, presence of ulceration or regression, and response to therapy, were found to be correlated with serum NEDD9 levels $(\mathrm{P}>0.05)$ (Table II).

Factors affecting survival. The median survival of all patients was 20.8 months (95\% CI: 10.7-30.9). The 1- and 2-year overall survival rates were 67.3 and $44.4 \%$, respectively. Truncal lesions $(\mathrm{P}=0.027)$, nodal involvement $(\mathrm{P}=0.08)$, multiple nodal involvement $(\mathrm{P}=0.047)$, metastasis $(\mathrm{P}<0.001)$, advanced metastasis $(\mathrm{P}<0.001)$, anemia $(\mathrm{P}<0.001)$, elevated erythrocyte sedimentation rate $(\mathrm{ESR})(\mathrm{P}=0.003)$ and failure to respond to chemotherapy $(\mathrm{P}=0.006)$ were found to be correlated with poorer survival (Table II). However, serum NEDD9 level did not appear to be of prognostic value for melanoma survival [hazard ratio $(\mathrm{HR})=1.142 ; 95 \% \mathrm{CI}: 0.588-2.217 ; \mathrm{P}=0.495$ ] (Table II; Fig. 1).

\section{Discussion}

The serum NEDD9 (also referred to as HEF1 and CAS-L) concentration in the 112 melanoma patients was found to be significantly higher compared with that in the healthy controls (median values: $3,784.02$ vs. $2,149.03 \mathrm{ng} / \mathrm{ml}$, respectively; 
Table II. Distribution and survival comparisons of serum NEDD9 levels on various patient/clinical parameters in patients with melanoma.

\begin{tabular}{lcc}
\hline & NEDD9 distribution & Survival \\
Parameters & P-value & P-value \\
\hline
\end{tabular}

Age, years

$<50 / \geq 50$

Sex

Male/female

0.88

0.76

Site of lesion

Axial/extremity

Histology

Nodular/non-nodular

Breslow thickness, mm

$\leq 4 />4$

Clark invasion level

I-III/IV-V

Ulceration

Yes/no

Mitotic rate (no. of

mitoses $/ \mathrm{mm}^{2}$ )

$0-2 / \geq 3$

Regression

Yes/no

TIL

Yes/no

Nodal involvement

Yes/no

Type of nodal involvement

Single/multiple

Metastasis

Yes/no

M1 status

$\mathrm{ab} / \mathrm{c}$

Serum LDH level

High/normal

Anemia

Yes/no

ESR

High/normal

Response to chemotherapy

Yes/no

0.047

0.001

0.001

0.001

NEDD9 expression

Low $<$ median $>$ high

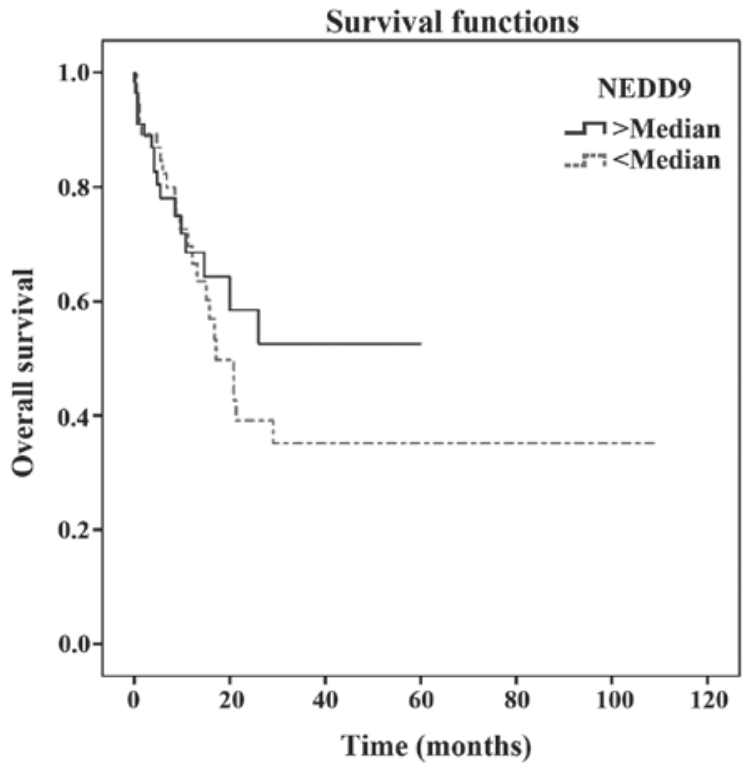

Figure 1. Survival curves in melanoma patients according to serum NEDD9 levels $(\mathrm{P}=0.495)$. NEDD9, neural precursor cell-expressed developmentally downregulated protein 9 .

$\mathrm{P}<0.001)$. NEDD9 concentration was not found to be correlated any of the following clinicopathological parameters: Site of lesion, lymph node involvement, stage, lactate dehydrogenase level, sex, histology, Breslow thiskness, Clark invasion level, presence of ulceration or regression and response to therapy $(\mathrm{P}>0.05)$. Truncal lesions $(\mathrm{P}=0.027)$, multiple nodal involvement $(\mathrm{P}=0.047)$, metastasis $(\mathrm{P}<0.001)$, advanced metastasis $(\mathrm{P}<0.001)$, anemia $(\mathrm{P}<0.001)$, elevated ESR $(\mathrm{P}=0.003)$ and failure to respond to chemotherapy $(\mathrm{P}=0.006)$ were correlated with poor survival. However, serum NEDD9 level had no prognostic effect on melanoma survival $(\mathrm{HR}=1.142 ; 95 \% \mathrm{CI}$ : $0.588-2.217 ; \mathrm{P}=0.495)$.

Cas scaffolding proteins (NEDD9/HEF1/CAS-L, BCAR1/p130Cas, EFS/Sin and HEPL/CASS4) play important roles in cell functions such as migration, proliferation and survival (1). Among these, BCAR1 has been associated with promotion of tumorigenesis, invasive behavior of the tumor and enhanced metastasis and, thus, unfavorable prognosis in breast cancer (10), whereas overexpression of NEDD9 has been correlated with glioblastoma and melanoma $(11,12)$. Epithelial-to-mesenchymal transition (EMT) is necessary for the invasive behavior of tumors, i.e., cells move more readily once their lateral attachments with adjacent cells are broken (1). E-cadherin normally acts as a cell-to-cell adhesion molecule, but it is downregulated during EMT, so the adherens junctions lose their stability and, thence, cells disconnect from one another (13). It has been demonstrated that NEDD9 or BCAR1 overexpression downregulate E-cadherin protein expression in cells, and conversely, E-cadherin expression is augmented when either NEDD9 or BCAR1, or both, are knocked down (1). This has been explained by Cas activation of lysosomal breakdown of E-cadherin through Src kinase. Similarly, NEDD9 depletion re-boosted E-cadherin expression, which was previously decreased by dioxin treatment that originally upregulated NEDD9 expression (14). E-cadherin loss from the cell surface by Cas proteins appears to be a

Bold print indicates statistical significance. NEDD9, neural precursor cell-expressed developmentally downregulated protein 9 ; TIL, tumor-infiltrating lymphocytes; LDH, lactate dehydrogenase; ESR, erythrocyte sedimentation rate. 
plausible explanation for the invasiveness of the tumors that express high levels of NEDD9.

The metastatic tendency of melanoma has already been associated with overexpression of NEDD9. Genomic modifications, such as chromosomal gain and loss, account for the development and/or invasiveness of several cancer types. Among these events, chromosome 6p gain has been particularly associated with several malignancies and their prognosis, including lymphoma, retinoblastoma, multiple myeloma and non-small-cell lung cancer (NSCLC), and the Nedd9 gene was found to be constantly upregulated in this amplified region in metastatic, but not in primary, melanoma cells excessively expressing the NEDD9 protein, which promotes metastasis in melanoma (7,12). Furthermore, NEDD9 knockdown resulted in inhibition of proliferation and invasion of melanoma cells; thus, continued NEDD9 expression was found to be necessary for melanoma cells to invade and metastasize (12). These studies demonstrated that NEDD9 in association with the RAS-RAF pathways increased the metastatic potential of primary non-transformed melanocytes and dormant melanoma cells (12).

Similarly, Rozenberg et al discovered overexpression of NEDD9 in metastatic melanoma cells in a murine model; however, interestingly, they also stated that NEDD9 lentiviral overexpression did not confer a metastatic ability on non-metastatic primary cells (15). This result supports the hypothesis that NEDD9 overexpression alone is not sufficient for tumorigenesis and invasiveness, but rather cooperation with other mechanisms impairing either checkpoints or apoptosis is required. However, downregulation of the Nedd9 gene (rather than its overexpression) was found in several studies, under suitable conditions, to be associated with increased invasiveness and metastasis $(16,17)$; furthermore, when significantly overexpressed, NEDD9 facilitates apoptosis and mitotic defects that activate checkpoints resulting in cell cycle dysregulation (18). All these data support the hypothesis that cells must be exposed to some genetic pre-alterations in conjunction with NEDD9 overexpression for proliferation, invasiveness and metastasis (3). Another study, although the underlying mechanism was not fully elucidated, revealed that increased activity of the inhibitor of $\beta$-catenin and T-cell factor/lymphoid enhancer factor caused a reduction of the NEDD9 level; this, in turn, resulted in less Rac1-GTP signaling, which is a positive regulator of mesenchymal movement, and it concurrently produced more Rho/ROCK-driven amoeboid movement of melanoma cells, which displayed an enhanced capacity for invasion and metastasis as a result of transformation to rounder and more motile shapes (19).

In their study, Lee et al suggested that the N-terminal truncated protein stimulated tumor growth and it may be used as a biomarker to predict the metastatic potential of various cancers, such as hepatocellular carcinoma and neuroendocrine cancers (20). That study demonstrated that, after being transported into the nucleus, the $\mathrm{N}$-terminal truncated protein co-functions with histone deacetylase 1/2 to increase Nedd 9 gene expression; in addition, by referring to the study by Kim et al (12), the significant role of NEDD9 in promoting melanoma invasiveness and metastasis was stressed (20).

The interaction between NEDD9 expression and cancer has been also investigated in other cancer types, including lung, breast and gastrointestinal cancer and glioblastoma. Since epidermal growth factor receptor (EGFR) expression and activation in NSCLC have long been reported and EGFR has already been affirmed as a treatment target, studies have been focused on possible molecular associations between EGFR and integrins regarding cellular invasion and metastasis. Since NEDD9 is a key protein of $\beta 1$-integrins and operates under a stringent association with EGFR, their association was specifically investigated. It was observed that tyrosine phosphorylation of NEDD9 was affected by overexpression of active EGFR without requiring integrin stimulation, and NEDD9 promoted migration and invasion of cells, thus facilitating NSCLC metastasis, whereas its expression in the primary tumor was found to be strongly associated with poor recurrence-free and overall survival (21). As reported by prior studies, the pro-metastatic role of NEDD9 in lung cancer was explained by its ability to induce EMT through FAK activation and the inverse correlation between NEDD9 and E-cadherin expression in lung cancer was also pointed out (22). It was successfully demonstrated that NEDD9 knockdown resulted in inhibition of migration, invasiveness and metastasis of lung cancer.

In agreement with the studies on other types of cancer, gastrointestinal cancers were also reported to be affected by elevated expression of NEDD9. Several studies reported the association between elevated NEDD9 expression and increased metastasis and poor prognosis in gastric cancer (23-26), pancreatic ductal adenocarcinoma (27), hepatocellular carcinoma (also in patients with early-stage disease and normal $\alpha$-fetoprotein levels) (28), and colorectal cancer (29).

The present study, conversely, demonstrated that serum NEDD9 levels were not associated with any of the poor prognostic variables for melanoma, and did not affect metastasis or survival. This lack of effect of NEDD9 on the prognosis of our patients may be attributed to the small number of the patients and the retrospective design of the study, and the results may have also been affected by the fact that we analyzed data that were collected over a short period of time. However, serum NEDD9 level was found to be a diagnostic factor for melanoma. Based on these results, taken together with the results reported by other studies, it is strongly believed that serum NEDD9 is of predictive and prognostic value in melanoma, as well as in other malignancies, and serum NEDD9 expression may be proven to be one of the predictive factors and a potential therapeutic target in melanoma. However, further investigation is required to prove this hypothesis.

\section{References}

1. Tikhmyanova N and Golemis EA: NEDD9 and BCAR1 negatively regulate E-cadherin membrane localization, and promote E-cadherin degradation. PLoS One 6: e22102, 2011.

2. Defilippi P, Di Stefano P and Cabodi S: p130Cas: A versatile scaffold in signaling networks. Trends Cell Biol 16: 257-263, 2006.

3. Singh M, Cowell L, Seo S, O'Neill G and Golemis E: Molecular basis for HEF1/NEDD9/Cas-L action as a multifunctional co-ordinator of invasion, apoptosis and cell cycle. Cell Biochem Biophys 48: 54-72, 2007.

4. Tikhmyanova N, Little JL and Golemis EA: CAS proteins in normal and pathological cell growth control. Cell Mol Life Sci 67: 1025-1048, 2010. 
5. O'Neill GM, Seo S, Serebriiskii IG, Lessin SR and Golemis EA Anew central scaffold formetastasis: Parsing HEF1/Cas-L/NEDD9. Cancer Res 67: 8975-8979, 2007.

6. Izumchenko E, Singh MK, Plotnikova OV, Tikhmyanova N, Little JL, Serebriiskii IG, Seo S, Kurokawa M, Egleston BL, Klein-Szanto A, et al: NEDD9 promotes oncogenic signaling in mammary tumor development. Cancer Res 69: 7198-7206, 2009.

7. Shagisultanova E, Gaponova AV, Gabbasov R, Nicolas E and Golemis EA: Preclinical and clinical studies of the NEDD9 scaffold protein in cancer and other diseases. Gene 567: 1-11, 2015.

8. Balch CM, Gershenwald JE, Soong S, Thompson JF, Atkins MB, Byrd DR, Buzaid AC, Cochran AJ, Coit DG, Ding S, et al: Final version of 2009 AJCC melanoma staging and classification. J Clin Oncol 27: 6199-6206, 2009.

9. Eisenhauer EA, Therasse P, Bogaerts J, Schwartz LH, Sargent D, Ford R, Dancey J, Arbuck S, Gwyther S, Mooney M, et al: New response evaluation criteria in solid tumours: Revised RECIST guideline (version 1.1). Eur J Cancer 45: 228-247, 2009.

10. van der Flier S, Brinkman A, Look MP, Kok EM, Meijer-van Gelder ME, Klijn JG, Dorssers LC and Foekens JA: Bcarl/p130Cas protein and primary breast cancer: Prognosis and response to tamoxifen treatment. J Natl Cancer Inst 92: 120-127, 2000.

11. Natarajan M, Stewart JE, Golemis EA, Pugacheva EN, Alexandropoulos K, Cox BD, Wang W, Grammer JR and Gladson CL: HEF1 is a necessary and specific downstream effector of FAK that promotes the migration of glioblastoma cells. Oncogene 25: 1721-1732, 2006.

12. Kim M, Gans JD, Nogueira C, Wang A, Paik JH, Feng B, Brennan C, Hahn WC, Cordon-Cardo C, Wagner SN, et al: Comparative oncogenomics identifies NEDD9 as a melanoma metastasis gene. Cell 125: 1269-1281, 2006.

13. Huber MA, Kraut N and Beug H: Molecular requirements for epithelial-mesenchymal transition during tumor progression. Curr Opin Cell Biol 17: 548-558, 2005.

14. Bui LC, Tomkiewicz C, Chevallier A, Pierre S, Bats AS, Mota S, Raingeaud J, Pierre J, Diry M, Transy C, et al: Nedd9/Hef1/Cas-L mediates the effects of environmental pollutants on cell migration and plasticity. Oncogene 28: 3642-3651, 2009.

15. Rozenberg GI, Monahan KB, Torrice C, Bear JE and Sharpless NE: Metastasis in an orthotopic murine model of melanoma is independent of RAS/RAF mutation. Melanoma Res 20: 361-371, 2010

16. Singh MK, Izumchenko E, Klein-Szanto AJ, Egleston BL, Wolfson $\mathrm{M}$ and Golemis EA: Enhanced genetic instability and dasatinib sensitivity in mammary tumor cells lacking NEDD9. Cancer Res 70: 8907-8916, 2010.
17. Minn AJ, Gupta GP, Siegel PM, Bos PD, Shu W, Giri DD, Viale A, Olshen AB, Gerald WL and Massagué J: Genes that mediate breast cancer metastasis to lung. Nature 436: 518-524, 2005.

18. Dadke D, Jarnik M, Pugacheva EN, Singh MK and Golemis EA: Deregulation of HEF1 impairs M-phase progression by disrupting the RhoA activation cycle. Mol Biol Cell 17: 1204-1217, 2006.

19. Domingues MJ, Rambow F, Job B, Papon L, Liu W, Larue L and Bonaventure $\mathrm{J}: \beta$-catenin inhibitor ICAT modulates the invasive motility of melanoma cells. Cancer Res 74: 1983-1995, 2014.

20. Lee TK, Murthy SR, Cawley NX, Dhanvantari S, Hewitt SM, Lou H, Lau T, Ma S, Huynh T, Wesley RA, et al: An N-terminal truncated carboxypeptidase $\mathrm{E}$ splice isoform induces tumor growth and is a biomarker for predicting future metastasis in human cancers. J Clin Invest 121: 880-892, 2011.

21. Kondo S, Iwata S, Yamada T, Inoue Y, Ichihara H, Kichikawa Y, Katayose T, Souta-Kuribara A, Yamazaki H, Hosono O, et al: Impact of the integrin signaling adaptor protein NEDD9 on prognosis and metastatic behavior of human lung cancer. Clin Cancer Res 18: 6326-6338, 2012.

22. Jin Y, Li F, Zheng C, Wang Y, Fang Z, Guo C, Wang X, Liu H, Deng L, Li C, et al: NEDD9 promotes lung cancer metastasis through epithelial-mesenchymal transition. Int J Cancer 134: 2294-2304, 2014

23. Zhang SS, Wu LH, Liu Q, Chen KS and Zhang XF: Elevated expression of NEDD9 is associated with metastatic activity in gastric cancer. Onco Targets Ther 8: 633-640, 2015.

24. Liu Y, Wang D, Zhao KL, Zhu JW, Yin HB, Wei YZ, Wu ZJ, Cheng GJ, Wang F, Ni F, et al: NEDD9 overexpression correlates with poor prognosis in gastric cancer. Tumour Biol 35: 6351-6356, 2014.

25. Shi R, Wang L, Wang T, Xu J, Wang F and Xu M: NEDD9 overexpression correlates with the progression and prognosis in gastric carcinoma. Med Oncol 31: 852, 2014.

26. Karabulut M, Alis H, Afsar CU, Karabulut S, Kocatas A, Oguz H and Aykan NF: Serum neural precursor cell-expressed, developmentally down regulated 9 (NEDD9) level may have a prognostic role in patients with gastric cancer. Biomed Pharmacother 73: 140-146, 2015.

27. Xue YZ, Sheng YY, Liu ZL, Wei ZQ, Cao HY, Wu YM, Lu YF, Yu LH, Li JP and Li ZS: Expression of NEDD9 in pancreatic ductal adenocarcinoma and its clinical significance. Tumour Biol 34: 895-899, 2013.

28. Lu P, Wang ZP, Dang Z, Zheng ZG, Li X, Zhou L, Ding R, Yue SQ and Dou KF: Expression of NEDD9 in hepatocellular carcinoma and its clinical significance. Oncol Rep 33: 2375-2383, 2015.

29. Li P, Zhou H, Zhu X, Ma G, Liu C, Lin B and Mao W: High expression of NEDD9 predicts adverse outcomes of colorectal cancer patients. Int J Clin Exp Pathol 7: 2565-2570, 2014. 